\title{
VOLATILIDAD MACROECONÓMICA Y DESIGUALDAD. VENEZUELA, 1960-2012. \\ EL CANAL DE LA INVERSIÓN ${ }^{1}$ \\ MACROECONOMIC VOLATILITY AND \\ INEQUALITY. VENEZUELA, 1960-2012. \\ THE INVESTMENT CHANEL
}

\author{
Carlos José Peña Parra \\ Instituto de Investigaciones Económicas y Sociales \\ Universidad Central de Venezuela \\ cpenaparra@gmail.com
}

\section{RESUMEN}

La volatilidad del crecimiento económico impacta negativamente sobre el bienestar social, se profundiza la mala distribución del ingreso y los niveles de consumo de las personas se ven reducidos. Lo más peligroso de este fenómeno es que la probabilidad de que los estratos de menores ingresos sean los más afectados es más alta debido a que la elasticidad ingreso de estos hogares es generalmente mayor. Así mismo, la volatilidad macroeconómica y el pobre desempeño económico ejercen un papel importante para explicar la desigualdad y la inestabilidad económica. En este contexto, la volatilidad de la tasa de inversión privada y el desempleo pueden dar una idea de los canales más importantes a través de los cuales se expresan los choques macroeconómicos y la volatilidad del crecimiento económico.

1 Este documento forma parte del proyecto de investigación del posdoctorado en ciencias sociales: Inestabilidad y desigualdad. Venezuela, 1960 - 2013. El papel de la volatilidad del crecimiento, Universidad Central de Venezuela. 
Dado lo anterior, el objetivo del presente trabajo es establecer la dinámica de la volatilidad macroeconómica y la desigualdad mediante el canal de la inversión en Venezuela para el periodo 1960 - 2012. La metodología la determina dicha dinámica, está basada en los modelos de vectores autorregresivos cointegrados, VARC, que permiten esbozar un modelo econométricamente bien especificado en presencia de variables no estacionarias. Esto implica que una formulación estocástica de los fenómenos económicos a través de un VARC permite abarcar, de forma general, la mayoría de las hipótesis económicas sin que se pierdan las propiedades estadísticas de los datos.

Los resultados validan que, a largo plazo, un aumento en la volatilidad de la inversión ocasiona un deterioro en la tasa de desempleo, lo cual podría estar generando un crecimiento en la desigualdad vía mercado de trabajo.

Palabras clave: inversión, volatilidad, desigualdad, desempleo

Clasificación JEL: C22, E24, E32

\begin{abstract}
The volatility of economic growth has a negative impact on social welfare, both because it deepens the bad distribution of income as well as the levels of consumption of persons they are reduced. The most dangerous of this phenomenon is that the probability that the lower income strata are most affected is higher, since elasticity - this household income is usually higher. Likewise, macroeconomic volatility and poor economic performance have an important role to explain inequality and economic instability. In this context, the volatility of the rate of private investment and unemployment can give an idea of the most important channels through which macroeconomic shocks and the volatility of economic growth are expressed.

Given the above, the objective of the present work is to establish the dynamics of macroeconomic volatility and inequality through the channel
\end{abstract}


of investment in Venezuela for the period 1960-2012. The methodology determine it the dynamics is based cointegrated vector autoregressive models VARC, allowing sketching a model well specified in the presence of nonstationary variables. This implies that a stochastic formulation of economic phenomena through a VARC allows encompassing, in general, most of the economic assumptions without that the statistical properties of the data lost.

Keywords: investment, volatility, inequality and unemployment

\section{INTRODUCCIÓN}

La volatilidad macroeconómica tiene importantes consecuencias, no solo económicas sino también sociales. Una de ellas está relacionada con las posibles repercusiones sobre el crecimiento económico y la inversión, en particular la privada. Es decir, una elevada volatilidad macroeconómica es perversa para la estabilidad económica y para la asignación eficiente de los recursos. El coeficiente de inversión privada debe crecer a una tasa constante, de tal manera que se pueda lograr un mayor crecimiento económico. Esto, porque a largo plazo el crecimiento económico y la inversión privada están correlacionadas positivamente. Sin embargo, si ese crecimiento es inestable igual que la inversión, sus consecuencias sobre el bienestar económico y social son perversas y generan más desigualdad e inseguridad económica.

Pudiera asumirse que la volatilidad macroeconómica y el pobre desempeño económico desempeñan un papel importante para explicar la desigualdad y la inestabilidad económica, aunque está claro que no pueden explicar por completo el comportamiento de las mismas. La volatilidad de la tasa de inversión privada y el desempleo pueden dar una idea de los canales más importantes a través de los cuales se expresan los choques macroeconómicos; no obstante, hay que decir que estas volatilidades dependen de la magnitud de los choques que recibe la economía.

En Venezuela, según los datos, el PIB real per cápita para el lapso comprendido entre 1960 y 2012 creció en promedio anual $0.6 \%$; en tanto 
que la volatilidad fue superior al crecimiento y se situó en 3.1 por ciento. La tasa de inversión privada (inversión privada/PIB) fue de $16.8 \%$ en promedio anual para igual periodo. Su variación porcentual se ubicó en $0.2 \%$ y la volatilidad de esta en 15.3 por ciento. Dichos datos evidencian los profundos desequilibrios e inestabilidad macroeconómica que ha padecido la economía venezolana. Como consecuencia de esto, los costos asociados en términos sociales han sido altos, entre ellos la volatilidad del desempleo y la pobreza. A tal situación hay que añadir la frecuencia de los choques externos, bien sean positivos o negativos, los cuales tienen efectos sobre la volatilidad del crecimiento. En este contexto, el objetivo del trabajo es establecer la dinámica entre la inversión privada, la desigualdad y la volatilidad macroeconómica en Venezuela para el lapso de 1960 a 2012.

La metodología se basa en los modelos VAR cointegrados VARC, que permite esbozar un modelo econométricamente bien especificado en presencia de variables no estacionarias. Esto implica que una formulación estocástica de los fenómenos económicos a través de un VARC permite abarcar, de forma general, la mayoría de las hipótesis económicas sin que se pierdan las propiedades estadísticas de los datos. Así, en términos generales, un VAR irrestricto bien especificado es un buen elemento analítico, pero al agregarle la peculiaridad de la cointegración, sus bondades econométricas se amplían.

El documento está estructurado de la siguiente manera. En la siguiente sección se presentan las aproximaciones teóricas; en la tercera, se intenta establecer la relación entre la volatilidad, inversión privada y desempeño económico; en la cuarta, la evidencia empírica a través de la metodología econométrica y, por último, se presentan las conclusiones.

\section{ASPECTOS TEÓRICOS}

\subsection{Inversión privada, crecimiento económico y desigualdad}

Si se parte de lo que plantea la literatura especializada (véase, por ejemplo, Jiménez y Manuelito, 2014) de que el crecimiento económico y la inversión, 
en particular la privada, están correlacionadas en el largo plazo, que el primero está en función de la acumulación de capital y que para lograr mayores tasas de crecimiento es necesario aumentar los coeficientes de inversión, entonces la importancia de la inversión es relevante. Dada esta premisa, se puede plantear que en la medida en que la inversión aumenta sostenidamente, será un factor esencial del crecimiento a largo plazo, esto, principalmente, por los efectos sobre la oferta y la definición de estructura económica, lo que confiere particular relieve a la acumulación de capital. Además de posibilitar la expansión de la capacidad productiva, existen otros factores favorecidos por el aumento de la inversión. En primer lugar, la inversión, en especial la privada, ${ }^{1}$ es un vehículo privilegiado para la incorporación del progreso técnico, que después se expresará en aumentos de la productividad del capital y trabajo, los cuales influirán en mayor crecimiento. En segundo término, es un mecanismo para el cambio estructural, ya que, a través de ella, se puede materializar la reasignación de recursos hacia sectores más dinámicos de la economía y mejorar los encadenamientos productivos, dos factores que posibilitan las ganancias de eficiencia, productividad y competitividad sistémicas de la estructura económica, esto es, aumentos en la productividad total de los factores.

Lo anterior implicaría que la inversión privada emerge así como el mecanismo mediante el cual se puede tener acceso a una nueva realidad económica, aquella en donde el consumo y bienestar guardan una relación más estrecha con las capacidades para producir bienes y servicios de valor agregado. Más importante aún, una realidad en donde los impuestos se convierten en un vínculo entre la capacidad del Estado para producir bienes públicos y las posibilidades del sector privado para crecer y desarrollarse. Estos vínculos, que resultan familiares no sólo en teoría sino también en la práctica económica de la mayoría de los países del mundo, sirven además como dispositivos de balance de poder fundamentales para el desarrollo. En este contexto, una precondición fundamental para la reducción de la

1 La inversión pública es un caso especial y cobra una especial importancia, ya que cumple un papel complementario de la inversión privada mediante la generación de externalidades necesarias para la obtención de rentabilidades en los proyectos privados. La ausencia de una eficiente infraestructura limita el crecimiento de la inversión privada y la sesga hacia sectores de enclave. Todo lo cual contribuye a un bajo crecimiento de las economías. 
pobreza es un patrón de crecimiento $^{2}$ y cambio estructural que genere empleo productivo, mejore los ingresos y contribuya al bienestar general de la población. Al respecto existe una amplia literatura que ha resaltado la relación inversa entre el crecimiento económico y pobreza. (Véase, por ejemplo, Ravallion, 1995,1997, 2003; Ferreira, Leite y Ravallion, 2007; Barrientos, Ramírez y Tabares, 2014, entre otros).

El empleo ${ }^{3}$ constituye un canal fundamental a través del cual el ingreso derivado del crecimiento puede compartirse ampliamente. Si las personas cuentan con empleos remunerados adecuadamente, pueden salir de la pobreza, participar en los esquemas de seguridad social que fortalecen su bienestar y mejorar su estatus educativo y de salud. En suma, el crecimiento centrado en el empleo ${ }^{4}$ puede tener un sólido efecto multiplicador en diversos aspectos. Sin embargo, el crecimiento en muchos países de bajos ingresos no ha sido sostenido y no ha logrado crear empleos.

La literatura ha mostrado que una elevada inestabilidad macroeconómica afecta negativamente a la distribución del ingreso. Consideraciones teóricas y empíricas indican que una buena parte de este impacto se canaliza por medio del mercado de trabajo, pues la elevada volatilidad del crecimiento económico es, entre otros, desfavorable para la generación de empleo de calidad y el acceso diferenciado a dichos puestos de trabajo (Weller, 2012). No sorprende, por lo tanto, que las fases de desempleo típicamente inciden en pérdidas de calidad de empleo, específicamente de ingresos laborales, incluso si los afectados encuentran rápidamente un nuevo empleo (véase, por ejemplo, Bucheli y Furtado, 2002; Herrera e Hidalgo, 2003; entre otros).

En estecontexto, la inversión, en especial la privada, refleja la dinámica macroeconómica de un país y es un indicador clave del comportamiento de

2 La hipótesis del patrón del crecimiento, HPC, afirma que las diferencias en el crecimiento económico, así como las diferencias regionales, afectan la reducción de la tasa de pobreza, independientemente de la tasa global del crecimiento del PIB.

3 El funcionamiento del mercado laboral impacta directamente en las decisiones y bienestar de los individuos.

4 El trabajo es uno de los factores de producción que contribuyen al crecimiento económico, y sus características inciden en las pautas y la sostenibilidad económica de este. Por otro lado, una expansión de la producción más allá de cierto umbral suele estimular la creación de nuevos puestos de trabajo y un aumento del nivel de empleo. 
una economía. La necesidad de aumentar la participación del sector privado en la formación de capital total es de hecho más aceptada. No obstante, depender de una estrategia de crecimiento liderada por la inversión privada es difícil si esta inversión forma una proporción relativamente pequeña del PIB y si la tasa de inversión no es especialmente dinámica.

\subsection{Volatilidad del crecimiento, de la inversión y desigualdad}

La literatura empírica y teórica vincula directamente el crecimiento económico con la volatilidad. Se ha encontrado una relación negativa entre volatilidad y crecimiento económico y los conductos que van de una variable a otra no son de fácil identificación. En todo caso, la literatura que establece esta relación es muy amplia. Por ejemplo, para Venezuela puede verse Peña (2013), sin embargo, el trabajo clásico por excelencia es el de Ramey y Ramey (1995). Ellos mostraron evidencia de un vínculo negativo entre ambas variables. Un canal importante es la inversión, en particular, la privada. La evidencia muestra cierta centralidad en la marcada variabilidad de la inversión.

Como lo mencionan Larraín y Parro (2008), igual que existe un efecto directo entre el crecimiento de la inversión y el crecimiento del producto de largo plazo, también existe un efecto en el mismo sentido entre las fluctuaciones del producto y la volatilidad de la inversión. La inversión total de una economía juega un papel muy importante en la determinación del crecimiento de largo plazo, donde la tasa de retorno del capital es clave en las decisiones de acumulación del mismo. Por lo tanto, en la medida en que la volatilidad del crecimiento de la inversión se mantenga bajo control, también podría reducirse la volatilidad del crecimiento del Producto Interno Bruto.

Autores como Peña $(2005,2008)$ señalan que la volatilidad macroeconómica tiene efectos negativos sobre la inversión. Esta representa un mayor nivel de inseguridad, lo que significa que los inversionistas reacios a asumir riesgos exigirán una mayor rentabilidad esperada. Asímismo, el comportamiento de la inversión privada es un factor muy importante por 
considerar, y puede verse desde varias ópticas. Una de ellas está en los avances recientes en el análisis del comportamiento de la inversión, los cuales plantean un conjunto de elementos que la diferencian sustancialmente de las decisiones de ahorrar. De igual manera, algunas teorías recientes (véase Caballero, 1991; Abel y Eberly, 1994; Dixit y Pindyck, 1994; Manzano, 2002; Mendoza, 2003), se han centrado en las implicaciones de que la inversión física es en gran medida irreversible y por lo tanto puede posponerse. ${ }^{5}$ La característica de irreversible de la mayoría de los gastos de inversión en capital fijo señalada en la literatura más reciente (Pindyck, 1991 y Dixit, 1992, entre otros) implica la consideración de este tipo de gastos como un costo "hundido" (no recuperable).

La irreversibilidad ${ }^{6}$ de la inversión en bienes de capital fijo es consecuencia de su especificidad. Los bienes de capital son, en la mayoría de los casos, "industria-específicos" o más aún "firma-específicos", dentro de una misma industria. Para aquellos tipos de bienes que no poseen esas características (computadoras, equipo de transporte, etc.) se verifica de todos modos una irreversibilidad parcial originada de la diferencia entre sus precios de compra y reventa, aun siendo nuevos. Esto ha determinado enfatizar el papel de la incertidumbre en esta clase de decisiones, que no pueden ser revocadas sin incurrir en importantes costos. En tal situación, se puede esperar hasta disponer de toda la información necesaria acerca del entorno económico para invertir. En este sentido, se cuenta con la opción de posponer la inversión si las circunstancias son desfavorables. En situaciones de incertidumbre siempre existirá la renuncia a invertir.

5 A pesar del supuesto general que surge de la renuncia al riesgo y las consecuencias de la irreversibilidad de la inversión, por varias razones de orden técnico, en la práctica la teoría económica no cuenta con nada definitivo que establezca una clara relación entre inversión y volatilidad macroeconómica. Como la teoría no puede resolver el tema, se acude entonces a la evidencia empírica.

6 Si la decisión de invertir en capital fijo debe tomarse en un contexto incierto, en donde la información llega gradualmente y, además, dicha oportunidad no desaparece si no se realiza inmediatamente, el problema no se reduce a decidir si se invierte o no, sino también a cuándo hacerlo. Bajo estas 21 condiciones "esperar" tiene un valor positivo, dado que se podrá obtener mayor información con el tiempo sobre el desempeño futuro del proyecto. Dicho valor deberá ser comparado con el sacrificio de los ingresos corrientes por postergar el emprendimiento del proyecto. 
La volatilidad de la inversión privada tiene consecuencias negativas, si se considera que los individuos son adversos al riesgo. Adicionalmente, si los retornos al capital son decrecientes, ${ }^{7}$ la economía estaría mejor en un nivel de capital fijo, que oscilando entre dos niveles de capitales. Es decir, aun en el caso de que los individuos no sean adversos al riesgo, una inversión menos volátil es mejor (Manzano, 2002).

Estudios empíricos de corte transversal con data agregada son hasta cierto punto más abundantes. Hausmann y Gavin (1995) reportan una asociación negativa entre un índice de volatilidad macroeconómica, el cual combina el PIB real y la volatilidad de la tasa de cambio real y el ratio inversión/GDP, con información de países en desarrollo. En contraste, Bleaney (1996) encuentra que esa medida de volatilidad afecta adversamente el crecimiento económico en los países en desarrollo, pero no a la inversión agregada. Resultados similares son obtenidos por Ramey y Ramey (1995), quienes también usan la inversión agregada. Adicionalmente, Hausman y Gavin (1995) plantean que la volatilidad genera fluctuaciones en las tasas de retorno, por lo que diversificar la inversión es difícil en este contexto. Así, al reducir la volatilidad, será más claro para los agentes económicos decidir dónde invertir.

Otros estudios, en especial Aizenman y Marion (1995, 1996, 1999), reportan una relación negativa entre varios indicadores de inestabilidad económica tales como: la volatilidad de los términos de intercambio, de la inflación y del tipo de cambio real y la inversión privada. Ellos, además, demuestran que esas medidas de volatilidad contribuyen significativamente a explicar el comportamiento de la inversión privada

No obstante, más allá de todo lo planteado, una inversión volátil puede tener efectos negativos sobre la desigualdad y la pobreza, aumentándola. La alta volatilidad macroeconómica afecta negativamente la inversión, por lo tanto el crecimiento económico, y hace más difícil la reducción de pobreza. Desafortunadamente la falta de dinamismo en la inversión privada parece ser una descripción bastante exacta cuando el comportamiento de la misma es volátil. En general, las explicaciones para esta característica han enfatizado diversos canales.

7 Esto es, cada unidad adicional de capital rinde menos que la anterior. 


\section{Volatilidad, inversión privada y desempeño económico}

\subsection{Dinámica y comportamiento de la inversión privada}

El desempeño macroeconómico en Venezuela no ha sido del todo eficiente, dado por un crecimiento económico inestable y una dinámica de la inversión, en particular la privada, con una tendencia secular al estancamiento. En la literatura económica la importancia de la inversión privada para el crecimiento económico ya es una idea ampliamente aceptada. Peña (2010).

Infortunadamente esta falta de dinamismo parece ser una descripción bastante exacta del comportamiento de la inversión privada en Venezuela durante las últimas décadas. En general, las explicaciones para dicha característica de la economía venezolana han enfatizado diversos canales. Las razones son varias. Entre ellas, Valecillos (1989) señala que, como suele ocurrir con las variables fundamentales de la vida económica, no es posible en uno o dos factores principales la explicación del curso descendente y critico seguido por la inversión privada. Mas aún cuando se trata de un fenómeno altamente sensible a las influencias políticoinstitucionales, psicológicas, tecnológicas o económicas -en un sentido estricto-, y cuando, como en el caso de Venezuela, se han experimentado situaciones que conllevan alteraciones sustanciales en la estructura de la economía y en la orientación de la política económica.

En las siguientes dos gráficas se muestra el comportamiento y dinámica de la inversión privada. En la gráfica 1 se presenta la tasa de crecimiento de dicha variable. La particularidad que muestra la variable es su inestabilidad y fuerte volatilidad a lo largo del periodo de estudio. Muchas pueden ser las razones, una parte de ellas ya fueron mencionadas en el párrafo anterior; sin embargo, esta variable es muy sensible a lo que se denomina clima de inversión, el cual es afectado por dos variables fundamentales: incertidumbre y política económica. (véase Penfold, 2002; Peña, 2009). En la gráfica 2 se presenta la inversión privada como porcentaje del PIB, tmfbk, la cual muestra una tendencia secular al estancamiento, con marcadas fases de crecimiento y desaceleración. Como se puede ver, 
hasta 1978 la inversión privada presenta un crecimiento sostenido, acorde con las condiciones macroeconómicas, sociales y políticas existentes en el país. A partir de ahí, el descenso ha sido más drástico. La inversión privada doméstica como porcentaje del PIB viene descendiendo desde la década de los ochenta del siglo XX, tendencia que ha sido más pronunciada en los noventas. En efecto, la tasa de inversión privada se ubicó en los años noventa en $6.4 \%$, muy por debajo de la realizada en los años setenta, que fue de 16.9 porciento. En las primeras décadas del siglo XXI se registra una leve recuperación, pero no sostenible.

\section{Gráfica 1}

Tasa de crecimiento de la inversión privada. Venezuela 1960-2012

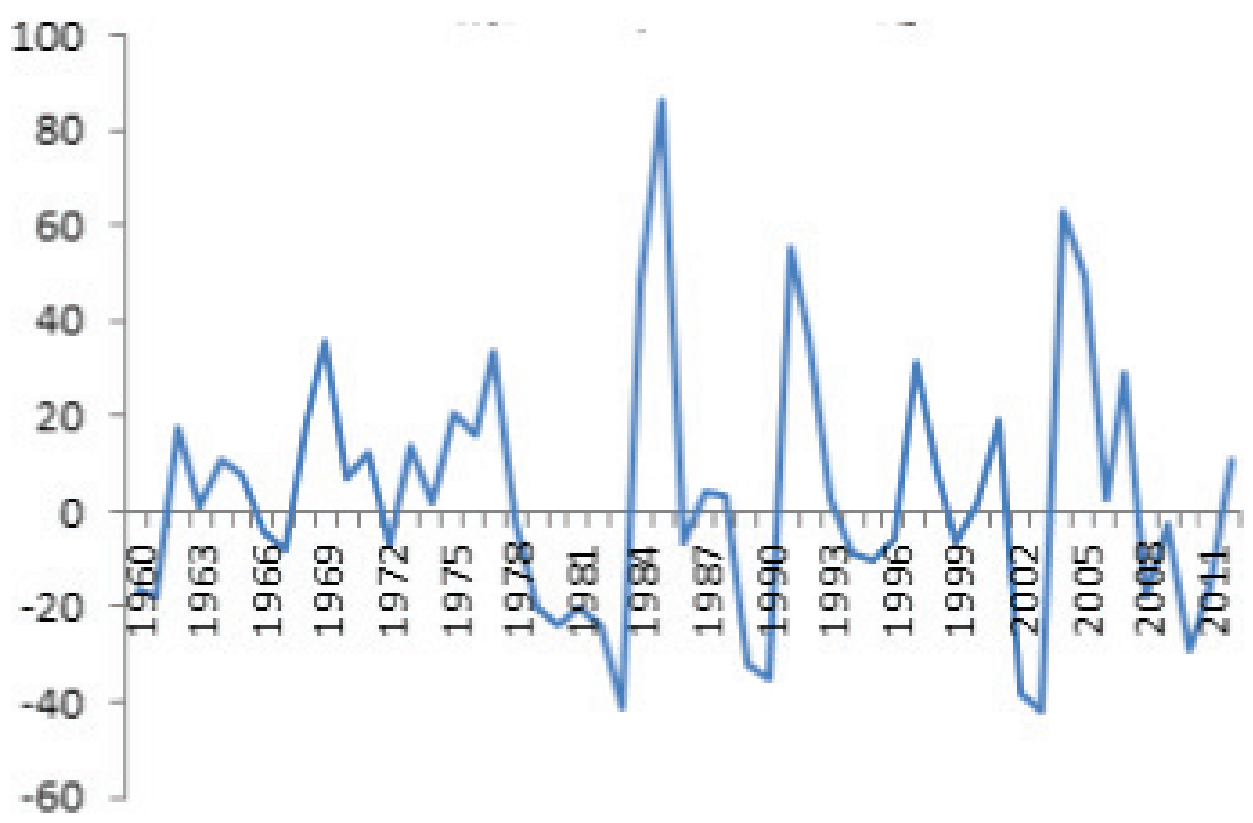

Fuente: Banco Central de Venezuela, BCV. Cálculos propios. 


\section{Gráfica 2}

Inversión privada como \% del PIB. Venezuela, 1960-2012

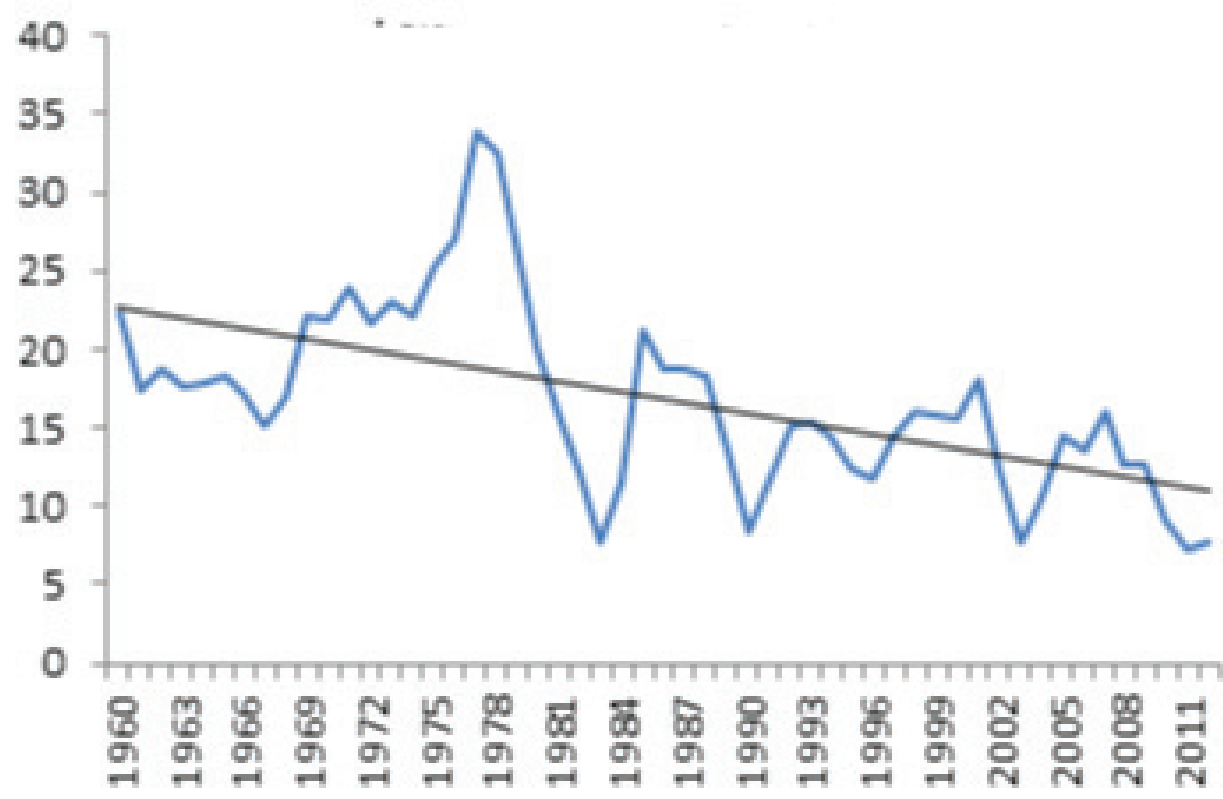

Fuente: Banco Central de Venezuela, BCV. Cálculos propios.

Lo que muestran la gráficas anteriores es el profundo deterioro en la dinámica y comportamiento de la inversión privada. Muchas son las causas que lo determinan: choques, tanto internos como externos, bien sean positivos o negativos, las diferentes crisis que ha padecido el país, políticas, sociales, institucionales, bancarias, cambiarias, etc.; sin embargo, como se plantea en la sección de los aspectos teóricos, hay un elemento que podría estar ejerciendo un efecto devastador en la inversión privada: la volatilidad del crecimiento o macroeconómica.

\subsection{Relación entre el crecimiento económico e inversión privada}

Si se parte de la idea que existe una relación positiva entre el crecimiento económico y la inversión, en particular la privada, entonces el desarrollo 
exitoso depende en gran medida de la iniciativa privada para producir e invertir en un país.

En la gráfica 3 se relacionan las tasas de variación porcentual, tanto del PIB per cápita, como de la inversión privada, tmfbk. Como se percibe, la relación entre ambas variables es positiva, sin embargo, la intensidad de ese trade off varía a lo largo del tiempo de estudio, al depender de cómo han sido los choques, tanto internos como externos. Así mismo, lo que refleja la gráfica es consistente con lo planteado a nivel teórico. Lo importante es distinguir que en la medida que la inversión se deteriora también lo hace el crecimiento.

Gráfica 3

Tasa de Inversión privada y PIB per cápita. Venezuela, 1960-2012

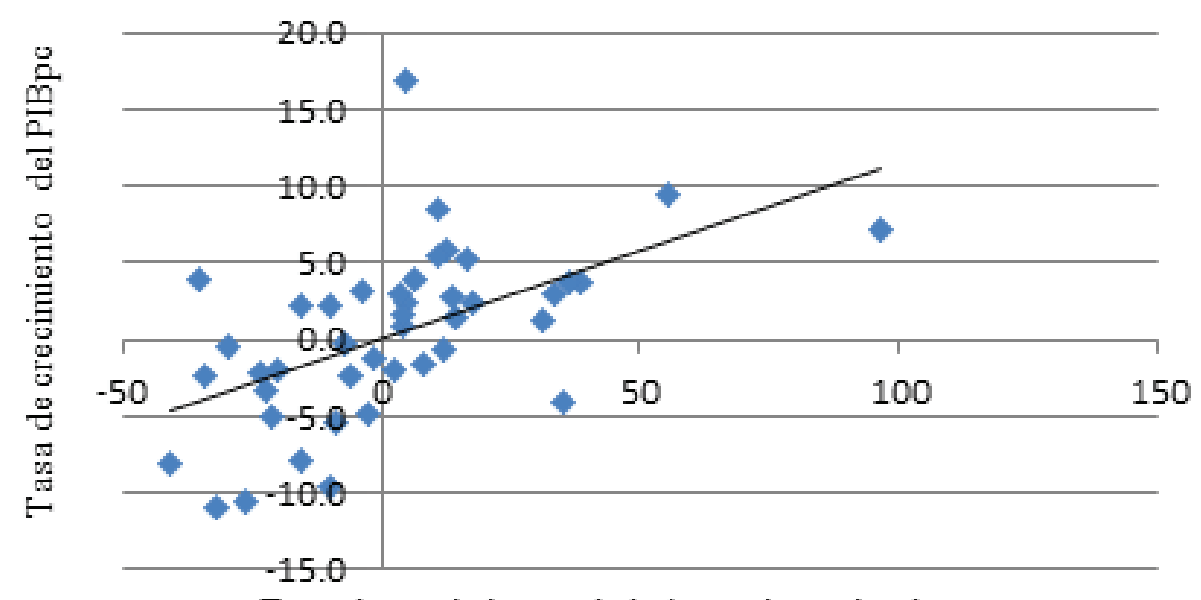

Tasa de crecimiento de la inversion privada

(\% del PIB)

Fuente. Banco Central de Venezuela, BCV. Cálculos propios.

Si bien es cierto que la inversión privada o pública no alcanza por si sola para explicar el crecimiento económico sostenido; en este sentido el capital es apenas uno de los factores que utiliza el proceso productivo, el cual depende de la disponibilidad y capacidad de la mano de obra y, en 
el largo plazo, de la tecnología. ${ }^{8}$ Según Santos (2006) en Venezuela tal consideración general tiene una importancia específica a la luz de la débil relación que históricamente presenta la inversión y el crecimiento. Dicha realidad no le resta importancia al argumento del clima de inversión y a la actividad empresarial privada. Desde este enfoque, la inversión privada se convierte en una condición necesaria, más no suficiente, para alcanzar un crecimiento económico sostenido.

\subsection{Volatilidad del crecimiento económico ${ }^{9}$ y de la inversión privada}

El crecimiento económico venezolano presenta un comportamiento inestable y volátil. La volatilidad del producto tiene efectos importantes sobre la inversión privada. La evidencia empírica muestra que la relación entre ambas variables tiende a ser negativa. En el cuadro 1 se presenta evidencia que pudiera sostener lo dicho anteriormente

En el cuadro en cuestión se muestra la dinámica del crecimiento económico, medido a través del Producto Interno Bruto per cápita, PIBpc, y la tmfbk. Para facilitar el análisis, el lapso de estudio se dividió en sub periodos, en donde se han presentado diversos acontecimientos. En este sentido, las cifras señalan que, en promedio, entre los años 1960 y 2012 el crecimiento económico, medido por la tasa de variación anual del PIBpc, se ubicó en apenas $0.6 \%$; en tanto que la tasa de inversión privada se situó en $16.8 \%$ por ciento. La situación anterior contrasta con la década de los 60 y 70 del siglo XX, donde se observa un crecimiento importante sustentado por una inversión privada. Esto fue posible, ya que dicho lapso estuvo caracterizado por condiciones macroeconómicas aceptables ${ }^{10}$ que permitieron este escenario.

8 Todo esfuerzo por generar un crecimiento sostenido debe estar sustentado, al menos, por incrementos positivos en capital físico y humano. De no ser así es inevitable que la adición de un factor, bajo la misma tecnología, termine ocasionando rendimientos marginales decrecientes.

9 En este trabajo la volatilidad de una variable se calcula a través de la desviación estándar de la tasa de crecimiento de dicha variable.

10 Este lapso estuvo caracterizado por un cuadro macroeconómico de rápido crecimiento económico, equilibrado interna y externamente. Adicionalmente, a la estabilidad cambiaria y bajos niveles de inflación se sumaron una moderada tasa de interés real positiva, lo que propició un clima de seguridad aceptable para los agentes económicos. Esto estaría acorde con los bajos 


\section{Cuadro 1}

Volatilidad macroeconómica y de la inversión privada Venezuela, 1960 - 2012

\begin{tabular}{|l|c|c|c|c|c|c|}
\cline { 2 - 7 } \multicolumn{1}{c|}{} & \multicolumn{5}{c|}{ Periodos } & Total \\
\cline { 2 - 7 } \multicolumn{1}{c|}{} & $1960-1969$ & $1970-1979$ & $1980-1989$ & $1990-1999$ & $2000-2012$ & $1960-2012$ \\
\hline tpibpc & 2.0 & 1.8 & -2.8 & -0.1 & 1.9 & $\mathbf{0 . 6}$ \\
\hline бtpibpc & 1.7 & 1.5 & 3.0 & 4.3 & 4.4 & $\mathbf{3 . 1}$ \\
\hline tmfbk & 18.4 & 25.7 & 15.8 & 13.5 & 12,1 & $\mathbf{1 6 . 8}$ \\
\hline mfbk & -1.1 & 2.2 & -0.7 & 4,0 & -2.3 & $\mathbf{0 . 2 1}$ \\
\hline omfbk & 9.8 & 12.5 & 19.9 & 14.9 & 18.7 & $\mathbf{1 5 . 3}$ \\
\hline
\end{tabular}

Fuente: Banco Central de Venezuela. Cálculos propios.

Nota: tpibpc: tasa de crecimiento promedio anual del PIBpc бtpibpc: desviación estándar de la tasa de crecimiento del PIBpc

tmfbk: inversión privada como \% del PIB (promedio anual) $m f b k$ : variación porcentual anual de tmfbk $\sigma t m f b k$ : desviación estándar de la variación porcentual de la $m f b k$

Antes de analizar lo que se señala en el cuadro 1 es importante aclarar que las cifras que se muestran representan el promedio anual del lapso que se indica.

De acuerdo con las cifras mostradas por décadas el crecimiento económico es pobre. La conclusión fundamental que surge es la excesiva volatilidad e inestabilidad que éste presenta, específicamente a partir de 1983. No obstante, estos resultados no son satisfactorios cuando se comparan con otras economías de la región.

Se evidencia que es muy alta la frecuencia de episodios de turbulencia que pueden caracterizarse como crisis, algunas de las cuales han desembocado en frenazos abruptos en los procesos de aceleración del crecimiento, cuando no en colapsos productivos, es decir, en disminuciones del valor absoluto del PIB per cápita. Un hecho también de importancia es que pueden ser frecuentes las turbulencias que podrían haber provocado cambios en la tendencia de largo plazo, en contraste con las perturbaciones transitorias que, por definición, exhiben una reversión a la media.

niveles de incertidumbre imperantes en el país. 
En cuanto al comportamiento de la inversión privada, se tiene que al observar los valores correspondientes al total del lapso de estudio, la tasa de inversión privada presenta una disminución importante a lo largo del mismo. También se observa que la tasa de variación porcentual de la inversión privada presenta una fuerte inestabilidad y sumamente volátil en tanto que la volatilidad de ésta inversión se incrementa. En cuanto a la volatilidad del producto y el coeficiente de inversión privada a PIB, la evidencia parece sugerir que a medida que la volatilidad se incrementa la $t m f b k$ disminuye. Es decir, pareciera que el vínculo entre ambas es negativo.

Tal comportamiento de la inversión está estrechamente relacionado con las fuertes oscilaciones en el ingreso petrolero, que provoca una trayectoria macroeconómica errática $\mathrm{y}$, sobre todo, impide que los agentes económicos puedan formar expectativas claras en cuanto al futuro económico a la hora de decidir dónde realizar sus inversiones. En consecuencia, la percepción de riesgo radica en la incertidumbre tanto en la orientación de la política económica como en la orientación política del gobierno para disminuir los efectos negativos de los choques petroleros, En la gráfica 4 se presenta esa relación.

\section{Gráfica 4}

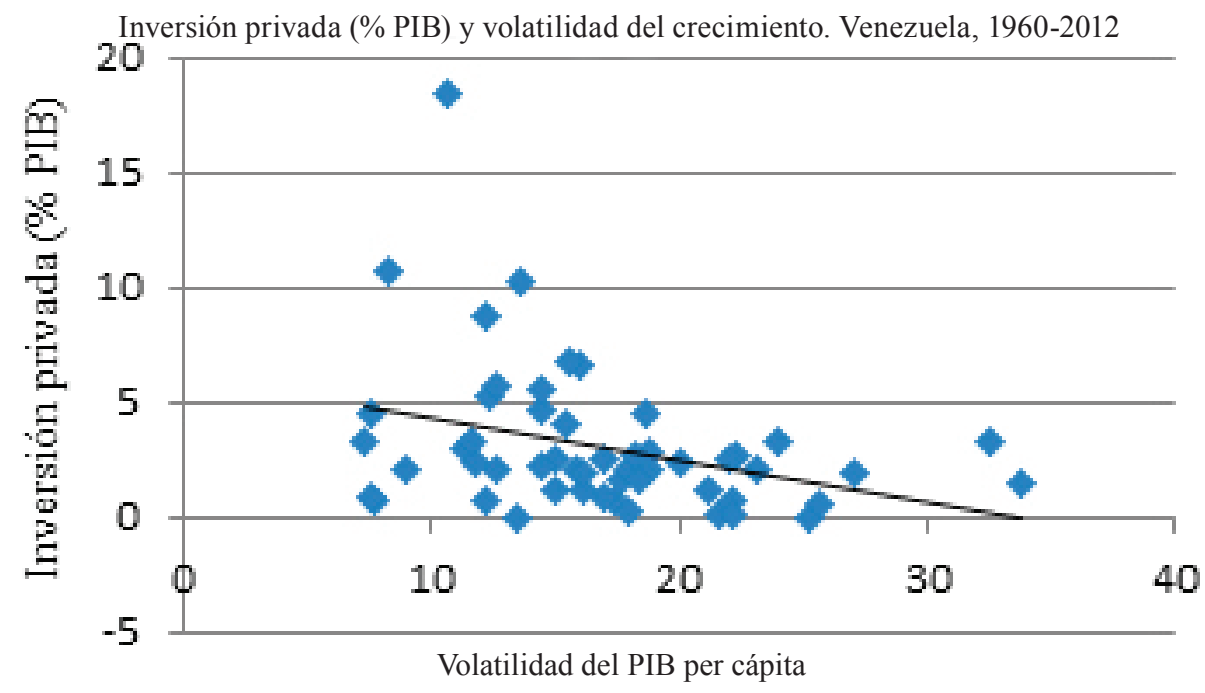

Fuente: Banco Central de Venezuela. Cálculos propios. 
Según lo mostrado en la gráfica, sí existe un nexo negativo entre las variables, sin embargo, la conexión no parece ser lo suficientemente fuerte.

\section{VOLATILIDAD MACROECONÓMICA, INVERSIÓN PRIVADA Y DESIGUALDAD. LA EVIDENCIA EMPÍRICA}

\subsection{Aspectos teóricos y metodológicos}

El punto fundamental es que la volatilidad del crecimiento económico incrementa la desigualdad a través de la inversión. En este sentido, la volatilidad no solo afecta la inversión agregada sino también la privada. Una tasa de inversión volátil impide que el crecimiento económico sea estable, ya que el comportamiento y dinámica de la inversión privada está ligado a los eventos macroeconómicos del lapso en estudio. En este sentido, la volatilidad del crecimiento económico juega un papel determinante en la volatilidad de la tasa de inversión. Peña (2005). Son diversos los factores que causan que el crecimiento económico sea volátil: monetarios, fiscales, choques externos, etc. (véase, por ejemplo, Cresta, 2012; Calderón y Schmitd-Hebbel, 2008, entre otros).

La hipótesis teórica sería en que si la inversión privada es volátil, esto podría estar implicando que la desigualdad también lo fueral o, en todo caso, aumentaría. El mecanismo a través del cual se manifestaría lo anterior sería el mercado de trabajo, particularmente con la tasa de desempleo. La primera impresión es que puede existir una relación positiva entre la volatilidad de la tasa de inversión y la tasa de desempleo, lo cual parece valido en una economía como la venezolana. Peña (2013) argumenta que a medida que la tasa de desempleo es más volatilil, se genera un efecto depresor sobre la productividad y los ingresos de la fuerza de trabajo. Esto puede implicar que, a medida que el desempleo aumenta o se hace más inestable, la desigualdad aumenta, ya que impacta en el bienestar vía ingresos laborales, lo que afecta negativamente la distribución del ingreso. En dicho sentido, la literatura ha mostrado que una elevada inestabilidad macroeconómica y de la inversión privada afecta negativamente la distribución del ingreso. (véase, por ejemplo, Gourdon, Maystre y De Melo, 2006). 
Consideraciones tanto empíricas como teóricas indican que buena parte de ese impacto se canaliza mediante el mercado de trabajo, ya que una elevada volatilidad macroeconómica es, entre otros aspectos, desfavorable para la generación de empleo de calidad y el acceso diferenciado a esos puestos de trabajo (Peña, 2005). Esto implica que la mayor o menor presencia de empleo de calidad ${ }^{11}$ estaría, en parte, determinado por el nivel y volatilidad de la tasa de inversión. Sin embargo, hay que ser cautos porque es posible no poder demostrar la hipótesis de que el aumento en los empleos de baja productividad este directamente relacionado con la volatilidad de la tasa de inversión.

El empleo es una de las variables que relaciona el bienestar social de la población con el crecimiento económico. No obstante, las señales del deterioro del mercado laboral en Venezuela parecen ser evidentes, pero también diversas. Una de ellas es el deterioro en la calidad del empleo, el cual está dado por el aumento de la informalidad, el incremento del empleo precario, ${ }^{12}$ el desempleo, etc. ${ }^{13}$

En este sentido, Zuñiga (2010: 355) señala:

Hoy en día el mercado laboral se encuentra en una grave situación que requiere ser atendida de inmediato: aumento del número de demandantes de puestos de trabajo; trabajadores con escasa capacitación; estructura productiva terciaria e improductiva; presencia de empleos precarios. Problemas que a su vez son retos a superar y que exigen la existencia de institucionalidad y políticas que apunten a ello.

11 Entendido esto como los puestos de jornada completa, con un horizonte temporal no predeterminado y que cuenten con algún grado de seguridad social.

12 La precariedad se manifiesta en un conjunto de insuficiencias que en unos casos obstaculiza y en otros imposibilita que los trabajadores mantengan unas condiciones de vida mínimas, en la medida en que deja de funcionar como un canal de integración social. Esta ausencia de calidad en el empleo se identifica en los siguientes aspectos: empleos con deficiencias salariales, inestables, con una relación desventajosa entre en ingreso obtenido y el número de horas trabajadas o trabajadores subutilizados.

13 Adicionalmente, existen un conjunto de regulaciones y restricciones laborales dadas, fundamentalmente, por un conjunto de leyes que han afectado el costo laboral o el costo de ajuste de la demanda de trabajo por parte de la empresa. Esas regulaciones afectan las decisiones de contratación y despido de las empresas privadas. 
La capacidad de absorción de trabajo productivo depende del crecimiento económico del país y este, a su vez, está sustentado en gran medida por la inversión, en particular la privada. En tal sentido, puede existir una correlación negativa entre la inversión privada y la tasa de desempleo.

\section{Cuadro 2}

Tasa de inversión privada y tasa de desempleo. Promedio anual Venezuela, 1960 - 2012

\begin{tabular}{|c|c|c|c|c|c|c|}
\cline { 2 - 7 } \multicolumn{1}{c|}{} & \multicolumn{5}{c|}{ Periodos } & Promedio \\
\cline { 2 - 7 } \multicolumn{1}{c|}{} & $1960-1969$ & $1970-1979$ & $1980-1989$ & $1990-1999$ & $2000-2012$ & $1960-2012$ \\
\hline tdes & 10.3 & 6.1 & 8.5 & 9.1 & 11.5 & 9.1 \\
\hline tmfbk & 18.4 & 25.7 & 15.8 & 13.5 & 12.1 & 16.8 \\
\hline informal & 42.6 & 37.6 & 40.7 & 46.9 & 47.8 & 43.1 \\
\hline tpibpc & 2 & 1.8 & -2.8 & -0.1 & 1.9 & 0.6 \\
\hline
\end{tabular}

Fuente: Banco Central de Venezuela, BCV. Instituto Nacional de Estadísticas, INE. Cálculos propios. Nota: tdes: tasa de desempleo; informal: tasa de informalidad; tmfbk: tasa de inversión privada; tpibpc: tasa de crecimiento del PIB per cápita

El cuadro 2 pone de manifiesto la incapacidad de la economía y, en especial de la inversión privada, para generar empleo de calidad. Se observa como la caída de la tasa de inversión, resultado de los profundos desequilibrios macroeconómicos y choques externos que provocan un clima de inversión adverso que la hace muy sensible a los riesgos, ha sido recurrente, lo que ha ocasionado un aumento en la tasa de desempleo e informalidad. Como señalan las cifras, la inversión privada como porcentaje del PIB es insuficiente, no sólo para generar un crecimiento sostenido, si no también para crear empleo de calidad. Según Guerra (2005) el aumento del desempleo guarda relación con el pobre desempeño de la economía venezolana. Así mismo, señala que la caída de la inversión privada se refleja en un desplome del empleo y, por consiguiente, en un aumento del desempleo. En consecuencia, el incremento del desempleo y de empleo de baja calidad puede estar asociado a la caída de la tasa de inversión. Esta situación hace que la inversión privada sea volátil, lo que causa efectos perversos en el mercado de trabajo (Peña, 2005). 
El función de lo anterior, la estrategia econométrica que se presenta se basa en la metodología desarrollada por Juselius (2006) del VAR cointegrado; ${ }^{14}$ VARC que permite esbozar un modelo bien especificado en presencia de variables no estacionarias. Loría, González y García (2009) explican que una formulación estocástica de los fenómenos económicos a través de un VARC ${ }^{15}$ permite abarcar, de forma general, la mayoría de las hipótesis económicas sin que se pierdan las propiedades estadísticas de los datos. Así, en términos generales, un VAR irrestricto bien especificado es un buen elemento analítico, pero al agregarle la peculiaridad de la cointegración sus bondades econométricas se amplían. En este sentido, Garrat et al (2006), exponen la orientación de los modelos VAR cointegrados:

- Hacer uso de la teoría económica para imponer restricciones con el fin de identificar las matrices $\alpha$ y $\beta$.

- La identificación precisa, obligatoriamente, no se alcanza con restricciones teóricas.

Una representación adecuada de un modelo de cointegración es a través de un modelo de corrección de errores, VEC. ${ }^{16}$ Varios elementos caracterizan este tipo de modelos, entre ellos: se asume que la teoría económica determina el comportamiento de largo plazo, que las fluctuaciones de corto plazo representan desviaciones con respecto a la relación de equilibrio de largo plazo, las nociones económicas de corto y largo plazo están unidas al concepto de estacionariedad. La representación de un VECM, conocido por sus siglas en inglés como vector error correction model sería:

14 Están basados en la especificación de los modelos VEC (VAR con corrección de errores).

15 Esta metodología permite incorporar la mayoría de los supuestos de un modelo teórico a un conjunto de restricciones estadísticas que pueden ser probadas a través de un modelo que cumpla satisfactoriamente los supuestos básicos de correcta especificación.

16 Los modelos VEC se diferencias de los modelos VAR en que emplean variables no estacionarias y, en este sentido, es capaz de captar elementos de gran importancia en el análisis de series de tiempo. 
Donde: $\Delta Y_{t}=\varphi_{0}+\varphi_{1} * t-\alpha \beta Y_{t-1}+\sum_{i=1}^{p-1} \Gamma_{i} \Delta Y_{t-1}+u_{t}$

$\mathrm{Y}_{\mathrm{t}}$ : Vector de variables endógenas $\mathrm{I}(1)$.

$\alpha \beta$ : Matrices que incluyen las relaciones de largo plazo y los coeficientes de ajustes al equilibrio de largo plazo.

$\Gamma_{\mathrm{i}}$ : Matriz con coeficientes asociados a los efectos dinámicos de corto plazo.

$\varphi_{0} \varphi_{1}$ : Vectores con coeficientes asociados al intercepto y la tendencia deterministica.

Según Sánchez y Zavarce (2013), la identificación de $\alpha$ y $\beta$, las cuales son matrices de $n$ variables que representan $r$ relaciones de cointegración y necesariamente debe cumplirse que $r \leq n$, requiere $\mathrm{r}^{2}$ restricciones. En este sentido, la estimación de Johansen $(1988,1991),{ }^{17}$ que presenta indudables ventajas frente a otras alternativas, identifica las matrices a y $\mathrm{b}$ imponiendo $r^{2}$ restricciones ateóricas.

Para Loría, González y García (2009) si $r=0$ implica que no existe relación de largo plazo entre las variables y que el VEC debe estimarse únicamente en primeras diferencias, con lo que solo estaría evaluando las variaciones de corto plazo de las $n$ variables involucradas, sin existir relaciones estables de largo plazo. Por otro lado, si hay un sistema de rango completo $r=n$, las variables son estacionarias, con o sin algún componente determinístico incorporado al vector de las variables. Por último, si $0<r<$ $n$ entonces existe un máximo de $n-1$ relaciones de cointegración.

17 Este método permite, respecto al procedimiento en dos etapas de Engle y Granger (1987), por ejemplo: i) contrastar el orden de integración de cada variable introducida en el modelo, ii) estimar los vectores de cointegración sin que la normalización del vector interfiera, iii) determinar directamente el número de las relaciones de cointegración existentes entre un conjunto de variables, iv) ganar en eficiencia al incorporar la información de corto plazo en la estimación de los parámetros de largo, v) realizar contrastes de hipótesis sobre los parámetros estimados. 


\subsection{Estacionariedad e integración}

Las pruebas de raíz unitaria se realizan para el logaritmo natural de las series en estudio, que son: tasa de desempleo, ltdes, volatilidad de la tasa de inversión privada, lotmfbk, tasa de inversión privada, ltmfbk, tasa de variación de la tasa de inversión privada, $l t f b k,{ }^{18}$ producto interno bruto per cápita, lpibpc, índice de precios al consumidor, lipc. Las pruebas incluyen un intercepto y una tendencia determinista para analizar si las variables son estacionarias en torno a dicha tendencia. Se recurre a las siguientes pruebas: Dickey-Fuller Ampliada (1979, 1981) o ADF; correcciones no paramétricas Phillips-Perron, PP, (1988). Sin embargo, Ramírez y Rodríguez (2008), Rivera y Toledo (2004) y Cámara (2006) consideran que las pruebas: DFGLS (Dickey-Fuller test with GLS detrending) o ERS (GLS) (véase Elliot, Rothenberg y Stock, 1996), son las de mayor potencia.

Para el contraste de ADF se utilizó el criterio de Akaike, AIC, para seleccionar el orden del proceso autorregresivo. El contraste de PP se realizó con la ventana espectral de Barlett. Los resultados se muestran en el cuadro 3.

\section{Cuadro 3}

Prueba de raíz unitaria

\begin{tabular}{|c|c|c|c|}
\hline & DFA & $P P$ & DF-GLS (ERS) \\
\hline Variable & $P$-valor & $P$-valor & $P$-valor \\
\hline lpibpc & 0.9860 & 0.8960 & 0.2940 \\
\hline Alpibpc & 0.0006 & 0.0001 & 0.0000 \\
\hline ltmfbk & 0.0996 & 0.1377 & 0.0890 \\
\hline Dltmfbk & 0.0001 & 0.0000 & 0.0009 \\
\hline lotmfbk & 0.1178 & 0.6785 & 0.9673 \\
\hline
\end{tabular}

18 Generalmente se parte del supuesto de que la tasa de crecimiento de la inversión, en este caso la privada, es determinante de la tasa de crecimiento de la economía. La variable que se ha trabajado hasta ahora ha sido la inversión privada como porcentaje del PIB, sin embargo, esta puede resultar inapropiada para un ejercicio como el que se propone, por la siguiente razón: al ser la tasa de inversión fracción de dos variables procíclicas (inversión y PIB), la inestabilidad de la inversión podría no quedar plenamente reflejada en la tasa de inversión (inversión/PIB), lo que, a su vez, podría subestimar los efectos sobre la variable endógena. Por tal motivo parecia conveniente modificar la definición de la variable inversión; en consecuencia, se utilizó la tasa de variación porcentual de la relación inversión/PIB. 


\begin{tabular}{|c|c|c|c|}
\hline$\Delta l \sigma t m f b k$ & 0.0000 & 0.0007 & 0.0020 \\
\hline ltdes & 0.0874 & 0.4200 & 0.1475 \\
\hline Dltdes & 0.0000 & 0.0002 & 0.0000 \\
\hline lipc & 0.5388 & 0.6032 & 0.2679 \\
\hline$\Delta l i p c$ & 0.0001 & 0.0001 & 0.0000 \\
\hline
\end{tabular}

Fuente: Cálculos propios.

Nivel de significancia estadística: $1 \%$.

DFA: Dickey-Fuller Aumentado, PP: Phillips-Perron.

Hipótesis nula: ADF y PP: la variable tiene raíz unitaria.

DFA: DF-GLS Dickey-Fuller detrending.

Como se observa, la evidencia muestra que las variables en estudio son estacionarias en primera diferencia, según las pruebas utilizadas. Por lo tanto, las series son $\mathrm{I}(1)$.

\subsection{Estimación y resultados}

Una vez definidas las condiciones de las series incorporadas en el modelo VAR cointegrado se estima el sistema y se verifican los supuestos de ruido blanco sobre los residuales, así como las pruebas para confirmar que cumple con las propiedades de estabilidad. Según las diferentes pruebas realizadas no se rechaza la hipótesis nula. Es decir, se acepta que no hay autocorrelación (multiplicador de Lagrange); así mismo, existe normalidad en los residuos, lo que implica que se comportan bajo una distribución normal multivariada. En conclusión, se encuentra que los residuales cumplen con los requisitos de comportamiento de ruido blanco, también se demuestra que el VAR estimado es estable.

Una vez estimado el VAR y verificar que cumple con los supuestos, se procedió a realizar la prueba de cointegración de Johansen ${ }^{19}$ (1988).

19 Dado que la distribución asintótica del estadístico de razón de verosimilitud de dicha prueba depende del supuesto que se haga con respecto a la presencia de intercepto y tendencia determinística en la ecuación de cointegración es necesario especificar dicha característica, previo a la instrumentación de dicha prueba. 
Para el presente trabajo se asumió tendencia lineal en los datos e intercepto en la ecuación de cointegración. Es decir, se supuso que la ecuación de cointegración no presenta tendencia. Adicionalmente, se especifica un (1) rezago para los términos en diferencias por fuera de la ecuación de integración; el resultado se muestra en el cuadro 4.

\section{Cuadro 4}

Prueba de cointegración de Johansen

\begin{tabular}{|l|c|c|c|c|}
\hline \multirow{2}{*}{\multicolumn{1}{c}{ Hipótesis }} & \multicolumn{4}{|c|}{ Test de rango de cointegración no restringido de la Traza } \\
\cline { 2 - 5 } & Valor propio & Estadístico de la traza & Valor critico & P-valor** \\
\hline Ninguna & 0.494475 & 36.29337 & 29.79707 & 0.0078 \\
\hline Al menos una & 0.177598 & 9.00709 & 15.49471 & 0.3647 \\
\hline Al menos dos & 0.029216 & 1.18606 & 3.84147 & 0.2761 \\
\hline
\end{tabular}

\begin{tabular}{|c|c|c|c|c|}
\hline \multirow{2}{*}{$\begin{array}{c}\text { Hipótesis } \\
\text { nula }\end{array}$} & \multicolumn{4}{|c|}{ Test de rango de cointegración no restringido del máximo valor propio } \\
\hline & Valor propio & $\begin{array}{c}\text { Estadistico del max } \\
\text { valor propio }\end{array}$ & Valor critico* & $P$-valor** \\
\hline Ninguna & 0.494475 & 27.28627 & 21.13162 & 0.0060 \\
\hline Al menos una & 0.177598 & 7.82103 & 14.26460 & 0.3972 \\
\hline Al menos dos & 0.029216 & 1.18606 & 3.84147 & 0.2761 \\
\hline
\end{tabular}

Fuente: Cálculos propios, *0,05, **MacKinnon-Haug-Michelis (1999) P-values.

Como se aprecia en el cuadro 4, la prueba de la traza rechazó con un nivel de significancia de 5\% la existencia de cero ecuaciones de cointegración. Al mismo tiempo, no fue posible rechazar la hipótesis nula que imponen máximo 1 y 2 relaciones de cointegración. En consecuencia, esta prueba indicó que existe una ecuación de cointegración para las variables en estudio. La prueba del máximo valor propio permitió concluir también lo mismo.

De acuerdo con la prueba de Johansen, el rango de cointegración de las variables analizadas es $r=1$ bajo la especificación de intercepto en la ecuación de cointegración, tendencia lineal en los datos y un rezago en los términos VAR. Con esta información se estimó el modelo de corrección de errores, VEC cointegrado. 
El hecho de que se haya encontrado que dichas variables presentan el mismo orden de integración y que existe una ecuación de cointegración entre ellas, permite concluir que dicha relación de largo plazo existe y que es posible estimar un modelo de corrección de errores, VEC, que describa el comportamiento de las variables a lo largo del tiempo. Se estimó un VEC (1), lo cual permitió que estadísticamente fuera satisfactorio. Esto es, se acepta la hipótesis nula de normalidad, no autocorrelación y homocedasticidad en los residuos; así mismo, el VEC presenta estabilidad en sus parámetros. Desde el punto de vista económico potencia las características del sistema económico discutido con anterioridad. En el cuadro siguiente se muestran los resultados.

\section{Cuadro 5}

Estimación del vector de corrección de errores, VEC

\begin{tabular}{|c|c|c|c|c|c|}
\hline Variables del sistema & ltdes & lotmfbk & Ltmfbk & lpibpc & loipc \\
\hline $\begin{array}{l}\text { Componentes del vector de } \\
\text { cointegración estimado } \\
\beta\end{array}$ & 1 & $\begin{array}{l}-0.1537 \\
(0.0326) \\
{[-4.7226]}\end{array}$ & $\begin{array}{l}0.5434 \\
(0.0994) \\
{[5.4681]}\end{array}$ & $\begin{array}{l}0.5325 \\
(0.0970) \\
{[5.4879]}\end{array}$ & $\begin{array}{l}-0.0946 \\
(0.0336) \\
{[-2.8169]}\end{array}$ \\
\hline $\begin{array}{l}\text { Componentes del vector de } \\
\text { velocidad de ajuste estimado } \\
\qquad \alpha \\
\end{array}$ & $\begin{array}{l}-0.1134 \\
(0.0023) \\
{[-5.8521]}\end{array}$ & $\begin{array}{l}5.1034 \\
(2.5574) \\
{[1.9956]}\end{array}$ & $\begin{array}{l}0.3855 \\
(0.4319) \\
{[0.8926]}\end{array}$ & $\begin{array}{l}0.8255 \\
(0.2798) \\
{[2.9505]}\end{array}$ & $\begin{array}{l}0.1156 \\
(0.2383) \\
{[0.4852]}\end{array}$ \\
\hline $\begin{array}{r}\text { Observaciones inc } \\
\text { Err } \\
\mathrm{T}-\mathrm{e}\end{array}$ & $\begin{array}{l}\text { didas } 40 \mathrm{~d} \\
\mathrm{r} \text { estándar } \\
\text { tadísticos }\end{array}$ & Ies & & & \\
\hline
\end{tabular}

Fuente: Cálculos propios.

Se observa que todos los coeficientes del vector de cointegración resultaron estadísticamente significativos a 1 y 5 por ciento.

El vector estimado resulta ser:

$$
\hat{\beta}^{\prime}=(1.0000-0.1537+0.5434+0.5325-0.0946)
$$

Nótese que dicho vector ha sido normalizado para que el primer coeficiente sea igual a 1, con el ordenamiento del vector de variables cointegradas se tiene que el equilibrio de largo plazo del sistema puede representarse como sigue: 


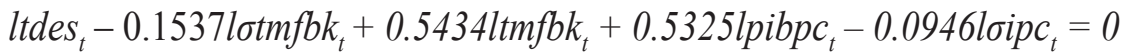

Es decir, la combinación lineal es una serie estacionaria. Ahora bien, esta se puede reescribir como

ltdes $_{t}=0.1537$ lotmfbk $k_{t}-0.5434 l t m f b k_{t}-0.5325 l p i b p c_{t}+0.0946 l \sigma i p c_{t}$

La expresión (4) describe las relaciones económicas de largo plazo. Dado esto, existe una relación estable a largo plazo entre la ltdes, ${ }^{20}$ la

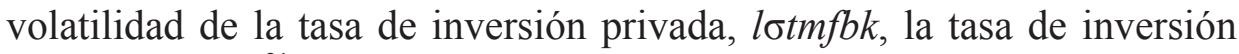
privada, ltmfbk, ${ }^{21}$ el lpibpc y la volatilidad de la tasa de inflación, løipc. Por otro lado, los resultados validan la relación negativa con ltmfbk y el lpibpc;

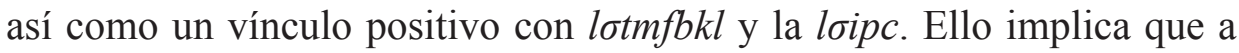

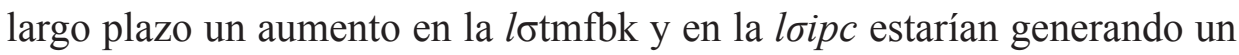
incremento en el en la tasa de desempleo. El impacto de la volatilidad de la inversión privada es la que más peso tiene. Por su parte, aumentos en la ltmfbk y en el lpibpc tenderán a disminuir la tasa de desempleo.

Con respecto al vector a estimado de velocidades de ajuste, se puede observar en el cuadro 5 que los coeficientes de la velocidad de ajuste de ltmfbky y loipc resultaron estadísticamente no significativos, lo que implica que estas ecuaciones no contribuyen a la restauración del equilibrio, por lo que, con los datos empleados, se sugiere que dichas variables serían débilmente exógenas. Por su parte, los coeficientes asociados a la velocidad de ajuste de la løtmfbk y lpibpc son estadísticamente significativos pero positivos, es decir, que no hay ajuste. En cuanto al coeficiente de la tasa de desempleo, ltdes, resultó con el signo correcto y estadísticamente significativo a 5\%, lo cual refuerza la hipótesis de la existencia de una relación de largo plazo entre las variables del modelo. En este caso, el valor del coeficiente es de 0.1134 , lo cual indica que el

20 La tasa de desempleo, tdes, se toma en esta sección como una variable proxy de la desigualdad.

21 Es importante mencionar que en el vector de cointegración se incluyeron las variables: tasa de variación porcentual de la inversión privada y la de la relación Inversión privada/PIB; ambas como medidas de inestabilidad; sin embargo, no resultaron estadísticamente significativas, por lo tanto no se muestran. En consecuencia, solo resultó estadísticamente significativa la relación inversión privada/PIB, que es la que se muestra. 
proceso de ajuste lleva tiempo en completarse, es decir, el ajuste de la ltdes es lento ante los desequilibrios y la inestabilidad que presentan las variables macroeconómicas, especialmente la inversión privada.

Para el caso analizado, que corresponde al periodo 1960 - 2012, han prevalecido los desequilibrios macroeconómicos ocasionados en parte por los choques externos, que han afectado sustancialmente la dinámica del mercado de trabajo en Venezuela,la, fuga de capitales, incertidumbre económica y política, inestabilidad y volatilidad macroeconómica, entre otros. En este sentido, el análisis lleva a los canales directos de la incidencia de las variables mencionadas sobre la tasa de desempleo y los efectos indirectos que se producen a través de los impactos de la política económica sobre el crecimiento económico.

\section{CONCLUSIONES}

El objetivo del presente documento fue establecer la dinámica entre la inversión privada, la desigualdad y la volatilidad macroeconómica en Venezuela para el lapso de 1960 a 2012. Fundamentalmente, resaltar el papel de la inversión privada en la desigualdad.

La falta de dinamismo dela inversión privada parece ser una descripción bastante exacta del comportamiento de esta variable en Venezuela durante las últimas décadas. En general, las explicaciones para esta característica de la economía venezolana han enfatizado diversos canales, entre otros, el de la volatilidad de crecimiento, que hace que la inversión también sea volátil. En todo caso, esta situación podría estar implicando que la desigualdad de igual manera sea volátil o bien, aumentaría. Esto implica que la mayor o menor presencia de empleo de calidad estaría, en parte, determinado por el nivel y volatilidad de la tasa de inversión.

En este contexto, la metodología utilizada fue la estimación de un modelo VAR cointegrado. De los resultados obtenidos se encontró que existe una relación de largo plazo, la cual es estable, entre la tasa de desempleo, tdes, la volatilidad de la tasa de inversión privada, lotmfbk, la inversión privada, ltmfbk, el producto per cápita, lpibpc y la volatilidad del a inflación, 
loipc. Así mismo, los resultados validaron la relación positiva entre la tdes,

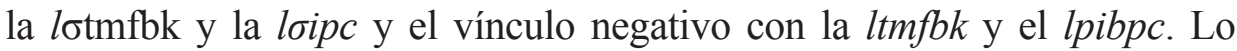
que implica que, a largo plazo, un aumento en la volatilidad de la inversión ocasionaría un deterioro de la tdes, e incrementaría el desempleo, lo que podría estar generando un crecimiento en la desigualdad vía mercado de trabajo. Por su parte, una expansión en la tasa de inversión privada tendrá un efecto inverso en la ltdes, lo cual ocasiona que esta disminuya.

Con respecto al vector estimado de velocidades de ajuste, se evidencia que el coeficiente de la tasa de desempleo, ltdes, resultó con el signo correcto y estadísticamente significativo a 5\%, lo que refuerza la hipótesis de la existencia de una relación de largo plazo entre las variables del modelo, principalmente con la tasa de inversión privada y su volatilidad. En este caso, el valor del coeficiente es de 0.1134 , lo cual indica que el proceso de ajuste lleva tiempo en completarse, es decir, el acople de la ltdes es lento ante los desequilibrios y la inestabilidad que presentan las variables macroeconómicas, especialmente la inversión privada.

\section{BIBLIOGRAFÍA}

Abel, A y J. Eberly. 1994. "A unified model of investment under uncertainty", NBER, Working paper series, núm. 4296.

Aizenman, J. y N. Marion. 1995. "Volatility, investment and disappointment aversion", NBER, Working paper series, núm.5386.

1996. "Volatility and the investment response" NBER, Working paper series, núm.5841.

1999. "Volatility and investment: interpreting evidence from developing countries", Economica, 66: 157 - 179.

Barrientos, M, S. Ramírez y E. Tabares. 2014. "El patrón de crecimiento económico en Colombia", Perfil de Coyuntura Económica, 24: 7 - 31 .

Bleaney, M. 1996. "Macroeconomic stability, investment and growth in developing countries", Journal of Development economics, 48: $461-477$. 
Bucheli, M. y M. Furtado. 2002. "Impacto del desempleo sobre el salario: el caso de Uruguay", Desarrollo Económico, 42(165): 63 - 84.

Caballero, R.J. 1991. "On the sign of the investment - uncertainty relationship", American Economic Review, 81(1): 279 - 288.

Calderón, C y K. Schmidt-Hebbel. 2008. "Business cycles and fiscal policies: the role of institutions and financial markets", Banco Central de Chile, Documento de trabajo, núm. 481.

Cámara, N. 2006. Raíces unitarias y cambios estructurales en los flujos del comercio internacional, Universidad de Zaragoza (mimeo).

Cresta, J. 2012. "La volatilidad de la economía y el esquema cambiario: un análisis empírico", Observatorio de Economía Internacional, OBEI, Informe núm. 3.

Dickey, D. y W. Fuller. 1979. "Distribution of the estimators for autoregressive time series with unit root", Journal of the American Statistical Association, 74(336): 427-431.

Dixit, A (1992) "Investment and Hysteresis" Journal of Economic Perspective. Vol. 6, No 1, pp. $107-132$

Dixit, A y R. Pindyck. 1994. Investment under uncertainty, MIT Press.

Elliot, G., T. Rothenberg y J. Stock. 1996. "Efficient test for an autoregressive unit root", Econometrica, 64 (4): 813 - 836.

Ferreira, F., P. Leite y M. Ravallion. 2007. "Poverty reduction without economics growth? Explaining Brazil's poverty dynamics, 1985 2004", World Bank policy research working paper, núm. 4432.

Johansen, S. 1988. "Statical analysis of co integration vector", Journal of Economic Dynamics and Control, 12: 231 - 254.

. 1991. "Estimation and hypothesis testing of co integration vector in Gaussian vector autoregressive models", Econometrica, 59(6): 1551-1580.

Gourdon, J., N. Maystre y J. De Melo. 2006. "Openness, inequality and poverty: Endowments matter", Banco Mundial, Policy research working papers, núm. 3981.

Jiménez, J.L y S. Manuelito. 2014. "Inversión y crecimiento en América Latina 1980 - 2012: rasgos estilizados de la relación”, en J.A. Fuentes Night (comp.). Inestabilidad y desigualdad. La vulnerabilidad del crecimiento 
económico en América Latina y el Caribe, CEPAL/Cooperación española/Cooperación alemán, capítulo III, pp. 105 - 144.

Juselius, K. 2006. The cointegrated VAR models. Methodology and applications, Oxford University Press.

Garrat, A., K. Lee, M.H. Pesaran y Y. Shin. 2006. "Global and national macro econometric modeling: A long-run structural approach", Oxford University Press.

Guerra, J. 2005. "El desempleo: problema nacional", Revista Venezolana de Análisis de Coyuntura, 11(2): 336 - 339.

Hausmann, R. y M. Gavin. 1995. "Macroeconomic volatility in Latin America: Causes, consequences and policies to assure stability", Banco Interamericano de Desarrollo (mimeo).

1996. "Securing stability and growth in a shock prone region: The policy challenger for Latin America" IADB Research Department Working Papers, núm. 315.

Herrera, J. y N. Hidalgo. 2003. "Vulnerabilidad del empleo en Lima. Un enfoque a partir de encuestas de hogares", CEPAL, Financiamiento del Desarrollo, núm. 130.

Larraín, F. y F. Parro. 2008. “Chile menos volátil”, El Trimestre Económico ,3(299): $563-596$.

Loría, E., T.D. González y M. García Ramos. 2009. "La metodología VAR cointegrado, un modelo de crecimiento económico para México, 1988 - 2007”, Facultad de Economía, UNAM, México (mimeo).

Mackinnon, J.G., A. Haug y L. Michelis. 1999. "Numerical distributions functions of likelihood ratio test for cointegration", Journal of Applied Econometrics, 14(5): 563 - 577.

Manzano, O. 2002. Vulnerabilidad externa y volatilidad económica, en J. Andrade (comp.), Temas Críticos para América Latina. Corporación Andina de Fomento, pp. 57 - 79.

Mendoza, O. (2003) “¿Cuáles factores determinan la inversión?”. Departamento de Información. Boletín Económico. BCVOZ. № 9. Banco Central de Venezuela.

Penfold, M. 2002. Costo politico-institucional, costo Venezuela: opciones de politica para mejorar la competitividad, CONAPRI, CAF, Caracas. 
Peña, C. 2005. "Volatilidad macroeconómica e inversión privada. Venezuela, 1968 - 2002", Revista Venezolana de Análisis de Coyuntura. XI (1): 185 - 202.

. 2008. "Choques petroleros, incertidumbre e inversión privada. Venezuela, 1968-2007”, Perfil de Coyuntura Económica, 11: 51 - 74. 2009. "Incertidumbre, renta petrolera y clima de inversión en Venezuela. Aspectos generales", Revista Venezolana de Análisis de Coyuntura, XV(1): $43-60$.

2010. "Shocks exógenos, dinámica macroeconómica e inversión privada. Venezuela, 1968 - 2009”, Perfil de Coyuntura Económica 16: $171-189$.

2013. "Volatilidad macroeconómica, shocks macroeconómicos y empleo. Venezuela, 1970 -2012", Temas de Coyuntura, 68: 62 - 84.

Pindyck, R (1991) "Irreversible uncertainty and investment" Journal of Economic Literature. Vol. 29. № 3, pp. 1110 - 1148

Phillips, P. y P. Perron. 1988. "Testing for a unit root in time series regression", Biometrika, 75(2): 335 - 346.

Ramírez, D. y G. Rodríguez. 2008. Estructura de la tasa de desempleo en España por comunidades autónomas, XXXIII Simposio de Análisis Económico, Zaragoza (mimeo).

Ramey, G. y V. Ramey. 1995. "Cross-country evidence on the link between volatility and growth",American Economic Review, 85(5): 1138 - 1151.

Ravallion, M. 1995. "Growth and poverty: Evidence for developing countries in the 1980s", Economics Letters, 48: 411 - 417.

y S. Chen. 1997. "What can new survey data tell us about in the distribution and poverty?", World Bank Economic Review, 11(1): 357 - 820.

. 2003. "Measuring pro-poor growth", Economics Letters, 78(1): 93 - 99.

Rivera, J. y P. Toledo. 2004. "Efectos de la infraestructura pública sobre el crecimiento de la economía, evidencia para Chile", Estudios de Economía, 31 (1): 21 - 38.

Santos, M.A. 2006. La inversión privada y el empobrecimiento sostenido de Venezuela, en A. Barrios Ross et al (comps.). Venezuela: un acuerdo para alcanzar el desarrollo, Instituto de Investigaciones Económicas y Sociales. Universidad Católica “Andrés Bello”. Caracas, Venezuela. 
Sánchez, G y H. Zavarce. 2013. Modelos VAR cointegrados y proyección probabilística. Una aplicación para economías pequeñas, Stata Press.

Valecillos, H. 1989. Acumulación de capital y desigualdades distributivas en la economía venezolana, Ediciones INAESIN, Caracas.

Weller, J. 2012. “Crecimiento, empleos y distribución de ingresos en América Latina", Macroeconomía del Desarrollo, núm. 122.

Zuñiga A. G. 2010. "Los retos del mercado laboral venezolano: más empleo, menos precariedad", SIC, 728: 355 - 367. 